UDC 621.37/.39:621.59:621.382.32

\title{
TEMPLATE MODELING OF A P-CHANNEL MOSFET
}

\author{
V. N. Biryukov \\ Institute for Radio Engineering Systems and Control of Southem Federal University, \\ Nekrasovsky, 44, Taganrog 347900, Russia
}

The paper is received on February 3, 2019

\begin{abstract}
A new MOSFET model for liquid-helium temperatures is introduced for needs of analog circuits designers. This model is created by the replacement of certain parameters of the original physical compact model by the quotient of power series of a gate and drain voltages. As the initial models, we chose Shichman-Hodges model and a model of Shockley based on the gradual channel approximation and the degradation of hole mobility in longitudinal electric field. Padé functions are used to simulate the degradation of carrier mobility in a transverse electric field and to correct the current - voltage characteristics in the region of high drain voltages. All parameters of the template model were determined simultaneously by the method of least squares. The total number of parameters of the proposed template model is eight, which makes it possible to use conventional programs for their identification. The relative root-mean-square error of modeling of $I-V$ curves of the template model is reduced by at least three times compared with the same error of the original model.
\end{abstract} Keywords: field-effect transistor, MOSFET, modeling, template model, root-meansquare, characterization, liquid-helium temperatures.

\section{Introduction}

Most transistor models for circuit simulation are often constructed in terms of basic electrical circuit elements such as controlled sources, resistors, and capacitors, arranged in a specific equivalent circuit topology. These transistor models are sometimes known as "compact" models, although their complexity has grown rapidly over time to the point where several standard compact device models have over 100 parameters and scores of nonlinear equations [1]. 
Compact models have high accuracy only for certain technology for a specific range of temperatures. For non-standard conditions the table models are commonly used. Table model represents a set of numerical data ordered in the form of various array tables, as well as various methods of interpolation of these data. Table models can reduce the simulation time, there is no need to identify parameters for them, and they are very simple to develop.

One of the disadvantages of table models is the complexity of the consideration of temperature influence and the inability of adjusting other parameters, such as threshold voltage. A direct solution of this problem is to construct a table with dimensions equal to the number of controlled variables. However, this alternative is now completely unacceptable in terms of volume of stored data [2]. Therefore, different ways of combining table modeling are used with analytic descriptions.

For continuity of all derivatives a way was ultimately offered of representation table model of the FET as a nonlinear function named template whose parameters are determined by a series expansion in the gate voltage [3]. This template model as an ordinary table model coincides current-voltage characteristics at certain points.

Herein we suggest exploring as a template the simple compact model of MOSFET with a small number of parameters. Further, we propose to replace the expansion parameters in power series by Padé expansion. Quotient of power series enable to get rid of the higher derivatives growth in the approximation. In addition, we propose characterization the parameters of a simple model and parameters as Padé function together by least squares method using measured $I-V$ curves of MOSFET. The term modeling is used here to denote both template model development and characterization [4].

The aim of this work is to create a model almost as simple and accurate as a table one, but that would have at least one physical parameter, namely the threshold voltage. This feature allows the statistical analysis of MOSFET's parameters, that a table model doesn't allow.

Below we argue that proposed model of the MOSFET can be used successfully at helium temperatures at which the well-known models lose their accuracy [5]. In 
addition, this model can be used during the development of new technologies of FETs such as InGaAs and SiGe.

\section{Models of origin}

Depending on the region of operation the drain current of the device is given $y$ the following equation of the Shichman-Hodges model [A\&M6]

$$
I_{D}=\left\{\begin{array}{lr}
0, & \text { for } V_{G} \leq 0, \\
\beta\left(V_{G}-\frac{V_{D S}}{2}\right) V_{D S}\left(1+\lambda V_{D S}\right), & \text { for } V_{D S}<V_{G}, \\
\beta V_{G}^{2}\left(1+\lambda V_{D S}\right), & \text { otherwise }
\end{array}\right.
$$

where $V_{G}=V_{G S}-V_{T H}$ is the effective gate voltage; $\beta$ is the intrinsic transconductance parameter; $V_{T H}$ is the device threshold voltage; and $\lambda$ is the channel length modulation factor; $V_{G S}$ and $V_{D S}$ are gate-to-source and drain-to-source voltages simultaneously.

More advanced IGFET model [Bisdounis7] comprise mobility dependence on the longitudal electric field in channel of the device as

$$
I_{D}=\left\{\begin{array}{lr}
0, & \text { for } V_{G} \leq 0, \\
\frac{\beta}{1+V_{0}^{-1} V_{D S}}\left(V_{G}-\frac{V_{D S}}{2}\right) V_{D S}, & \text { for } V_{D S}<V_{D S A T}, \\
\beta V_{0} V_{G}, & \text { otherwise }
\end{array}\right.
$$

here $V_{0}$ is the voltage which specifies the effect of carriers' velocity saturation and is extracted from the device static characteristics. $V_{D S A T}$ is the device saturation voltage and is given by

$$
V_{D S A T}=V_{0} \sqrt{1+V_{0}^{-1} V_{G}}-V_{0}
$$

Substituting multiplier $1+\lambda V_{D S}$ from (1) into this model, we get

$$
I_{D}=\left\{\begin{array}{lr}
0, & \text { for } V_{G} \leq 0, \\
\frac{\beta}{1+V_{0}^{-1} V_{D S}}\left(V_{G}-\frac{V_{D S}}{2}\right) V_{D S}\left(1+\lambda V_{D S}\right), & \text { for } V_{D S}<V D_{S A T}, \\
\beta V_{0} V_{G}\left(1+\lambda V_{D S}\right), & \text { otherwise },
\end{array}\right.
$$


Model (3), in contrast to (2), takes into account the final output conductivity of the transistor at any $V_{D S}$. Equation (3), as shown in Fig. 1 and Fig. 2, more precisely indicate the boundary between the linear and saturation regions of the $I-V$ curves, as $V_{D S A T}$ in (3) is less then pinch-off voltage $V_{G S}-V_{T H}$ in (1). Therefore, it has less error in this case.

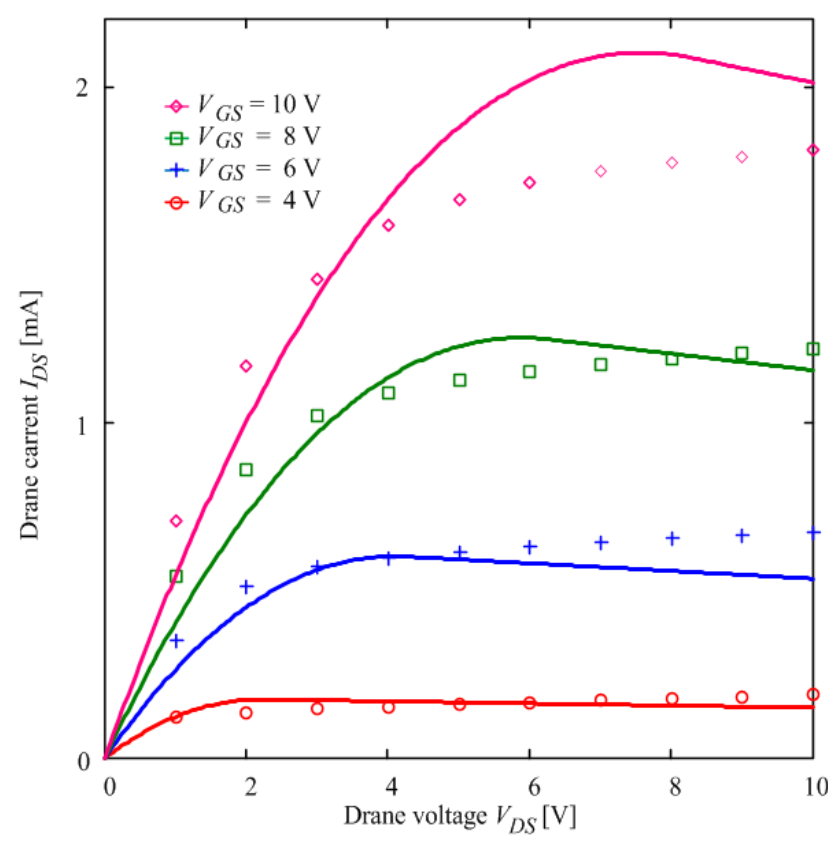

Fig. 1. Output characteristics of the model (1) of MOSFET with experimental data

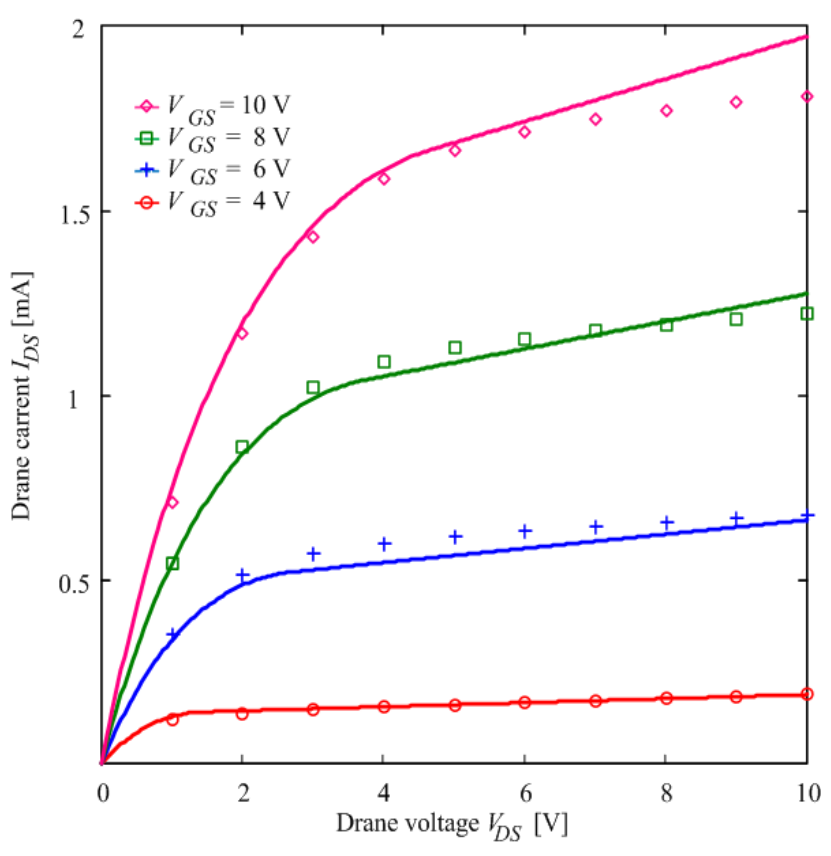

Fig. 2. Output characteristics of the model (3) of MOSFET with experimental data

\section{Template model}

The template for current state functions is given in [8] as follows:

$$
I_{D}\left(V_{D S}\right)=C+M \cdot\left(1+\lambda \cdot V_{D S}\right) \cdot \tanh \left(\alpha \cdot V_{D S}\right)
$$

where $\alpha, \lambda, M, C$ are the model (4) parameters. For each gate-to-source voltage $V_{G S}$, the four parameters $(\alpha, \lambda, M, C)$ in a parameterized behavioral function (1) are determined with special extraction routines. Then, the four parameters are fitted versus $V_{G S}$ using polynomial approximation.

The restrictions, imposed on the choice of the template, are its monotonicity and continuity. Thus, practically any compact model, whose parameters are determined by direct measurements, can be used as a template. In this paper we 
choose a simple $C_{1}$-continuous model of the MOSFET (3) as a template. Hereinafter, we use the replacement of the parameters $\beta$ and $\lambda$ by the quotient of power series to reduce the modeling error as

$$
\begin{gathered}
\beta=\beta_{0} \frac{\beta_{1} V_{G}}{\beta_{1} V_{G}+\beta_{2} V_{G}^{2}} \\
\lambda=\lambda_{0} \frac{\lambda_{1} V_{D S}}{\lambda_{1} V_{D S}+\lambda_{2} V_{D S}^{2}} \\
\lambda=\lambda_{0} \frac{\lambda_{1}\left(V_{D S}-V_{D S A T}\right)}{\lambda_{1}\left(V_{D S}-V_{D S A T}\right)+\lambda_{2}\left(V_{D S}-V_{D S A T}\right)^{2}} .
\end{gathered}
$$

The equations (5), (6) and (7) were chosen by trial and error approach. Their less successful or more complicated options are omitted here.

It should be noted that the Padé functions (5) and (6) represent a special case of the series relationship. Applications of the Padé functions for modeling allow to provide the monotonicity of $I-V$ curves unlike the power series expansion where the violation of the monotony of $I-V$ curves is possible [4].

In the present work the $p$-channel MOSFET with polysilicon gate was considered as the test sample for parametric identification. The experimental $I-V$ curves of the MOSFET test sample was measured at a temperature close to the boiling point of helium $(T=4.3 \mathrm{~K})$. The channel width and length $(W \approx 20 \mu \mathrm{m}$ and $L \approx 0.8 \mu \mathrm{m}$ ) of the test sample conformed to the sizes of transistors of cryogenic low noise amplifiers.

\section{Parametric identification of the models}

The forcefulness of the template modeling is largely determined by the effectiveness of parametric identification. It is convenient to determine physical parameters of the analytical MOSFET model by the least-squares minimum of the objective function as

$$
S(\mathbf{x})=\sum_{k=1}^{N}\left[\frac{I_{D}\left(V_{D S, k}, V_{G S, k}\right)-I_{k}}{I_{k}}\right]^{2}=\sum_{k=1}^{N} \delta_{k}^{2},
$$


where $\mathbf{x}=\left\{\beta, V_{T H}, \lambda, V_{0}\right\}$ is the vector of physical parameters; $\left\{I_{k}, V_{D S, k}, V_{G S, k}\right\}$, $k=1,2, \ldots, N$ is the experimental $I-V$ curve of MOSFET in tabular form, $N$ is the number of points of the $I-V$ curves.

Table 1. Parameters and errors of MOSFET's models

\begin{tabular}{|c|c|c|c|c|c|c|}
\hline \multirow{2}{*}{ Model } & \multicolumn{6}{|c|}{ Model's parameters } \\
\hline & $\beta\left[\mathrm{mA} \cdot \mathrm{V}^{-2}\right]$ & $\lambda\left[\mathrm{V}^{-1}\right]$ & $V_{0}[\mathrm{~V}]$ & $V_{T H}[\mathrm{~V}]$ & $s[\%]$ & $|\delta|_{\max }[\%]$ \\
\hline (1) & $\beta_{0}=0.07154$ & $\lambda_{0}=-0.01757$ & - & 1.7415 & 13.7 & 27 \\
\hline (3) & $\beta_{0}=0.1281$ & $\lambda_{0}=0.03978$ & 3.5865 & 2.259 & 4.30 & 8.4 \\
\hline (3)-(5) & $\begin{array}{l}\beta_{0}=0.16375 \\
\beta_{1}=0.44844 \\
\beta_{2}=0.02170\end{array}$ & $\lambda_{0}=0.03819$ & 4.1337 & 2.4683 & 2.72 & 9.0 \\
\hline (3)-(5)-(6) & $\begin{array}{c}\beta_{0}=0.1511 \\
\beta_{1}=0.4652 \\
\beta_{2}=0.01965\end{array}$ & $\begin{array}{l}\lambda_{0}=0.27651 \\
\lambda_{1}=0.11440 \\
\lambda_{2}=0.03528\end{array}$ & 2.7157 & 2.5488 & 1.40 & 4.6 \\
\hline (3)-(5)-(7) & $\begin{array}{c}\beta_{0}=0.17088 \\
\beta_{1}=0.31466 \\
\beta_{2}=0.024807\end{array}$ & $\begin{array}{l}\lambda_{0}=0.16289 \\
\lambda_{1}=0.14722 \\
\lambda_{2}=0.02091\end{array}$ & 2.7303 & 2.5707 & 1.26 & 4.6 \\
\hline
\end{tabular}

For solving curve-fitting problem we used Levenberg-Marquardt algorithm with the error control by variation of initials. To illustrate the effectiveness of the proposed method Table 1 shows the results of parameters extraction using different approximations $\beta\left(V_{G}\right)$ and $\lambda\left(V_{G}\right)$. The first row of Table 1 shows the parameters of the model (1). The second row presents the model parameters for the approximations (3). I-V curves for these rows are presented at Fig. 1 and Fig. 2 simultaneously. Further in the table the template model's data are given. The $I-V$ characteristics of the two options are shown in Figures 3 and 4. Accuracy of the experimental $I-V$ curves approximation in each case was estimated by value of the relative root-mean-square (RMS) error

$$
s=\sqrt{S\left(\mathbf{x}^{*}\right) / N},
$$

where $\mathbf{x}^{*}$ is the vector of parameters of the model corresponding to the minimum of the objective function (8). 


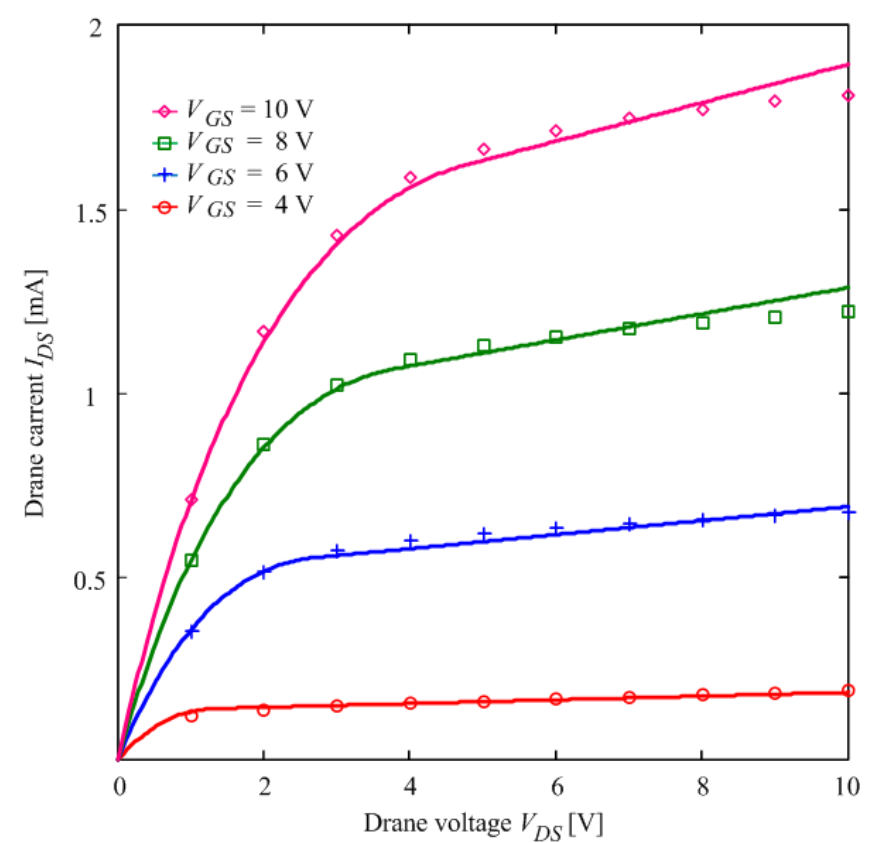

Fig. 3. Output characteristics of the template model (3)-(5) of MOSFET with experimental data

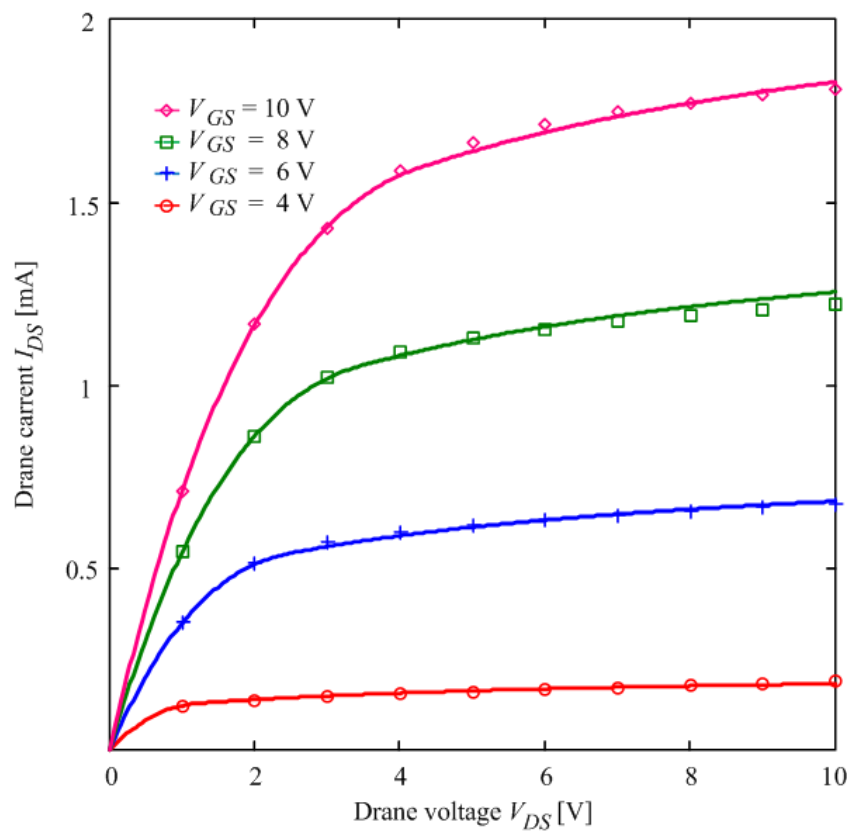

Fig. 4. Output characteristics of the template model (3)-(5)-(7) of MOSFET with experimental data

Used technique minimizes the RMS error. Unfortunately, the standard error does not give a complete picture of the quality of the approximation [9]. Therefore, along with the RMS error $s$ Table 1 gives the maximum relative error $|\delta|_{\max }$ also. Figures 5 and 6 show that during the procedure of descending the RMS error varies monotonically, but the maximum value of the current error varies no monotonically. Therefore, to obtain reliable results, it is desirable to rise the number of steps of descend until the value of the RMS error ceases to change. To improve the accuracy of the results, we can recommend the method of random descent [10].

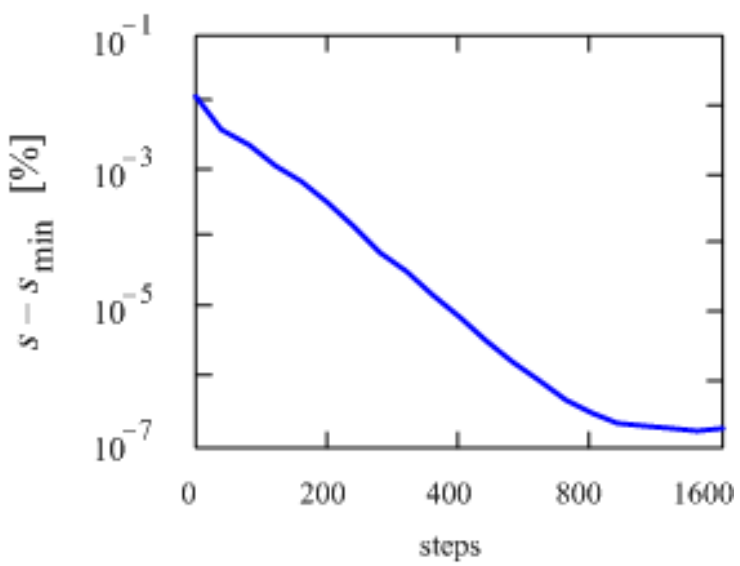

Fig. 5. RMS error versus steps of descend

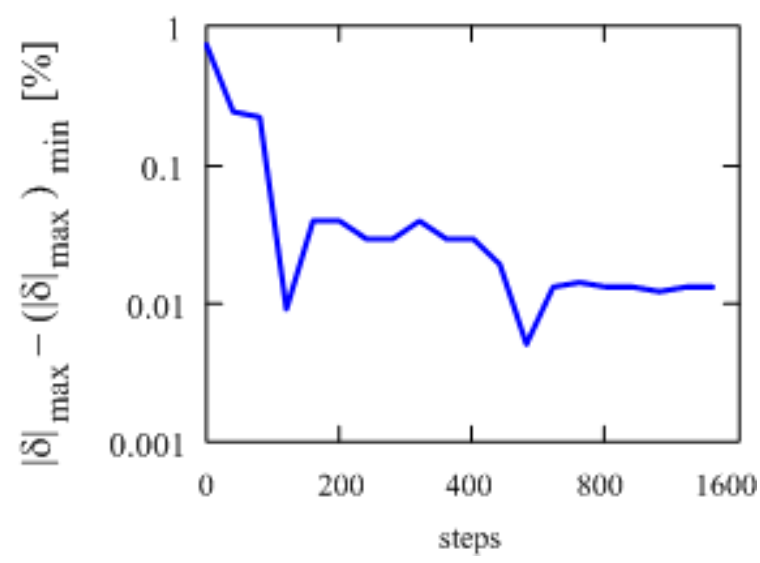

Fig. 6. $|\delta|_{\max }$ versus steps of descend 
With an increasing number of new factors in $\beta\left(V_{G}\right)$ and $\lambda\left(V_{D S}\right)$ root mean square error as expected decreases monotonically, but the maximum error $|\delta|_{\max }$ does not decrease monotonically. So, the refinement of the model (3) using (4) leads to a decrease in the mean square error $\sigma$, but does not guarantee a decrease in the maximum error $|\delta|_{\max }$. We have used more complicated approximations for $\beta\left(V_{G}\right)$ and $\lambda\left(V_{D S}\right)$ too, but a decree of the error was quite moderate. These results are not logged in the table.

The optimization problem in this case isn't stiff, so almost any conventional least squares method can be used to identify parameters. With an increase in the number of parameters, the effectiveness of these methods rapidly decreases. In fact, when the number of parameters is more than ten to twenty, the difficulties of the solution may prove insurmountable.

\section{Comparison of the models and discussion}

The Table 1 data indicate good agreement between measured and calculated $I$ $V$ characteristics. However, at designing the amplifier small signal characteristics output conductance $g=\partial I_{D} / \partial V_{D S}$ and transconductance $g_{m}=\partial I_{D} / \partial V_{G S}-$ are important too. A template model allows to get a very small error of the $I-V$ characteristics. However, it is not possible to get such a small error of small-signal parameters. The choice of Padé functions depends, as Figs 7 and 8 show, on a tradeoff between the error of the $I-V$ characteristics and the error of small-signal parameters.

The choice of the initial model and approximants is determined by many factors. First, the original model must provide the greatest possible accuracy. The initial model used in [11] in contrast to (3), is continuous with all its derivatives, but its accuracy is lower. Secondly, the models of $n$-channel and $p$-channel MOSFETs are basically different, since the mobility of holes and electrons depends on the electric field in different ways. This dependence is also determined by the crystal orientation of the substrate, and even depends on the orientation of the transistor on the substrate. The template model with some approximants may turn out to be numerically unstable (not robust). In addition, the choice of the approximants is largely affected by the need to limit the number of new parameters. 


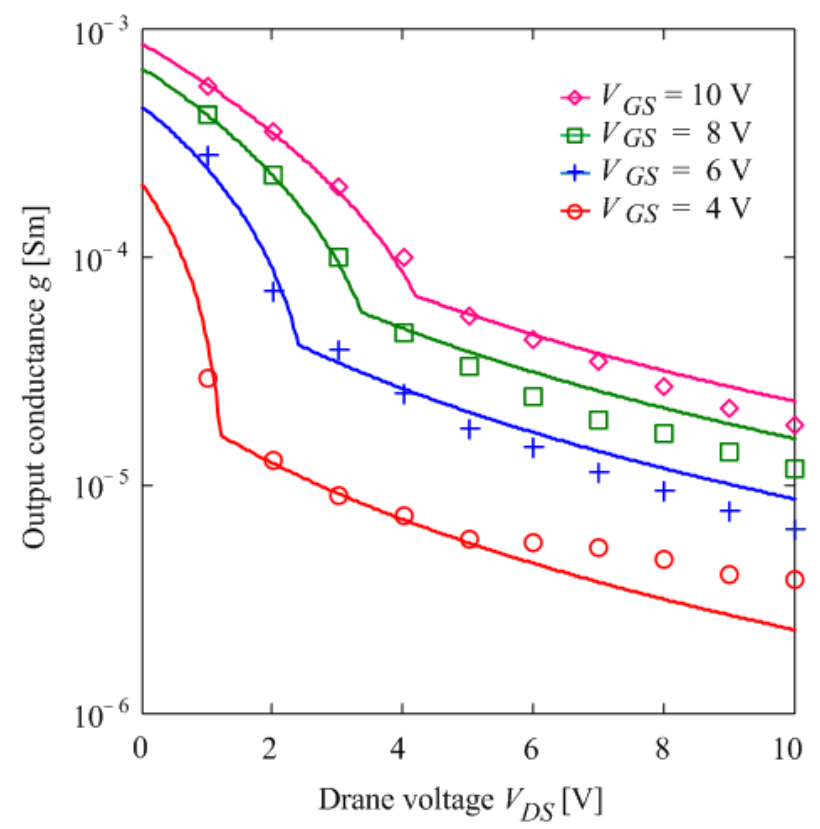

Fig. 7. Output conductance of the model Fig. 8. Output conductance of the model (3)-(5)-(6) with experimental data

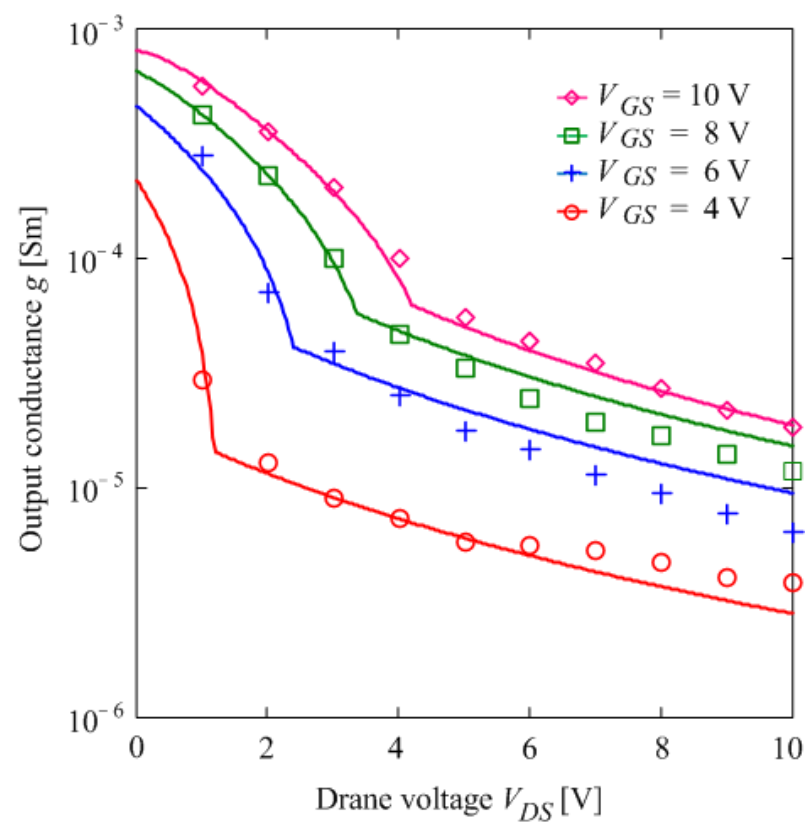

(3)-(5)-(7) with experimental data

It should be noted that the analytical model of the MOSFET can be obtained not only when the mobility of charge carriers depends on the longitudinal, but also on the transverse field [12]. The approximation of the mobility of carriers not in the expression for the intrinsic transconductance, but in the expression for the linear conductivity at an arbitrary point of the channel, it is possible to obtain a very high simulation accuracy. Unfortunately, an analytical model that takes into account the dependence of the mobility on the transverse and longitudinal components of the electric field cannot be obtained simultaneously.

The model (3) error increases at the weak inversion. However, it should be noted that this mode isn't used in low-noise amplifiers [13].

\section{Conclusions}

This paper has presented a new $\mathrm{C}_{1}$-continuous template model of MOSFET based on the original physical compact mode1. All parameters of an initial model except for threshold and characteristic voltages $V_{T H}$ and $V_{0}$ are represented by Padé approximant with effective gate and drain voltages as an argument. Approximation coefficients are determined in conjunction with the initial model parameters by the 
method of damped least squares. The template model isn't fully formal one as allows the threshold voltage extraction from voltage only measurements [14]. The threshold voltage in template model remains physical as in initial model. The initial physical model explored hitherto can be replaced by any simple one. This leads to template model flexibility.

Finally, it is important to note that when using the $L_{2}$ metric, it is expedient to provide information about the maximum error in modeling.

\section{References}

1. Advanced Design System 1.5. Circuit Components: Nonlinear Devices. Agilent Technologies, 2001.

2. V. V. Denisenko. A Table MOS Transistor Model Parameter Control. Russian Microelectronics, 2010, Vol. 39, No. 5, pp. 352-365.

3. S. Van den Bosch, L. Martens. Approximation of State Functions in Measurement-Based Transistor Model. IEEE Transactions on Microwave Theory and Techniques, 1999, Vol. 47, No. 1, pp. 14-17.

4. C. C. McAndrew. Practical modeling for circuit simulation. IEEE Journal of Solid-State Circuits, 1998, Vol. 33, No. 3, pp. 439-448.

5 J. Kan, S. Weifeng, and S. Longxing. A sub-circuit MOSFET model with a wide temperature range including cryogenic temperature. Journal of Semiconductors, 2011, Vol. 32, No. 6.

6. P. Antognetti, G. and Massobrio (Eds.) Semiconductor Device Modeling with SPICE. New York, McGraw-Hill, 1990.

7. L. Bisdounis, S. Nikolaidis, O. Koufopavlou, C. E. Goutis. Switching response modeling of the CMOS inverter for sub-micron devices. 1998 Proceedings of the conference on Design, automation and test in Europe, pp. 729-737.

8. S. Van den Bosch, and L. Martens. Approximation of State Functions in Measurement-Based Transistor Model. IEEE Transactions on Microwave Theory and Techniques, 1999, Vol. 47, No. 1, pp. 14-17. 
9. V. V. Denisenko. The Accuracy and Validity of the Simulation of VLSI MOS Transistors. Russian Microelectronics, 2009, Vol. 38, No. 4, pp. 273-278.

10. A. M. Pilipenko, V. N. Biryukov. Efficiency improvement of the random search algorithm for parametric identification of electronic components models. 2016 International Siberian Conference on Control and Communications (SIBCON). 12-14 May 2016.

11. V. N. Biryukov, A. M. Pilipenko. Measurement-based MOSFET model for helium temperatures. 2015 IEEE East-West Design \& Test Symposium (EWDTS). 26-29 Sept. 2015.

12. V. N. Biryukov. The effects of the transversal field dependence of carrier mobility on the validity of the gradual channel approximation in insulated-gate fieldeffect transistors. Journal of Communications Technology and Electronics, 1974, Vol. 19, No. 12, pp. 98-100.

13. V. N. Biryukov, A. M. Ponomarev, N. L. Tsipis. Investigation of the CurrentVoltage and Noise Characteristics of Field-Effect Transistors with An Insulated Gate at Low Temperatures. Radioelectronics and Communications Systems, 1986, Vol. 29, No. 11, pp. 108-111.

14. O. F. Siebel, M. C. Schneider, C. Galup-Montoro. MOSFET threshold voltage: Definition, extraction, and some applications. Microelectronics Journal, 2012, No. 43, pp. 329-336.

\section{For citation:}

V. N. Biryukov. Template modeling of a p-channel MOSFET. Zhurnal Radioelektroniki - Journal of Radio Electronics. 2019. No. 2. Available at http://jre.cplire.ru/jre/feb19/11/text.pdf

DOI 10.30898/1684-1719.2019.2.11 